\title{
Influência da intervenção fisioterapêutica na função motora grossa de crianças com paralisia cerebral diplégica: estudo de caso
}

\section{Influence of physiotherapeutic intervention in the thick motor function of children with diplegic cerebral palsy: case study}

\author{
Rutiméia Tagliavini Vedoatol; Amanda Rodrigues Conde'; Karina Pereira ${ }^{2}$ \\ ${ }^{1}$ Graduada em Fisioterapia - Uniara/SP \\ ${ }^{2}$ Docente em Fisioterapia - Uniara, Doutora em Fisioterapia - UFSCar/SP. \\ ENDEREÇO PARA CORRESPONDÊNCIA \\ Rutiméia Tagliavini Vedoato \\ Av. 28 de Agosto, $n^{\circ} 2100$ - B. Alto \\ 15990-000 - Matão - SP [Brasil] \\ E-mail: \\ rutimeia@ig.com.br
}

\begin{abstract}
RESUMO
O objetivo, neste estudo, foi verificar, quantitativa e qualitativamente, a influência da intervenção fisioterapêutica na habilidade motora grossa de crianças com paralisia cerebral diplégica. Participaram desta pesquisa duas crianças do sexo masculino. $\mathrm{O}$ instrumento utilizado na avaliação quantitativa foi a Gross Motor Function Measure. A avaliação qualitativa teve como base a análise descritiva postural nas posturas sentada e em pé e nas funções andar, correr e pular. Os métodos fisioterapêuticos utilizados foram: conceito neuroevolutivo Bobath, cinesioterapia e integração sensorial, associados às atividades lúdicas. A intervenção fisioterapêutica foi realizada duas vezes por semana, com duração de 50 minutos, totalizando 32 sessões durante quatro meses. Após intervenção, verificou-se melhora, quantitativa e qualitativa, tanto na postura sentada quanto nas funções de andar, correr e pular dos participantes. Constatou-se também que se não houve grande avanço quantitativo, na postura em pé. Em termos qualitativos, a evolução foi significativa.
\end{abstract}

Descritores: Descrição postural; Gross Motor Function Measure; Habilidade motora grossa; Intervenção fisioterapêutica; Paralisia cerebral diplégica.

\begin{abstract}
The objective of this study was to verify quantitatively and qualitatively the influence of the physiotherapic intervention in the thick motor ability in children with diplegic cerebral palsy. Two male children had participated of this study. The instrument used in the quantitative evaluation was Gross Motor Function Measure. The qualitative evaluation was based on the postural descriptive analysis on the seated and stood up postures and the walking, running and jumping functions. The physiotherapies' methods were: neuroevolutive concept Bobath, kinesioterapia and sensorial integration, associated to the playful activities. The physiotherapy intervention was carried through twice per week, a time period of fifty minutes, totalizing 32 sessions during 4 months. After intervention it was verified quantitative and qualitative improvement in the seated posture and in the functions of walking, running and to jump of the participants. In the stood up posture it did not have quantitative advance, however they had evolved qualitatively.
\end{abstract}

Key words: Diplegic cerebral palsy; Gross Motor Function Measure; Physiotherapy intervention; Postural description; Thick motor ability. 


\section{INTRODUÇÃO}

A atuação da fisioterapia nos programas de intervenção precoce, de educação e de reeducação psicomotora vêm apresentando grandiosos resultados na evolução neuro-sensório-motora de crianças atípicas ${ }^{1,2,3}$, principalmente quando as intervenções fisioterapêuticas são iniciadas precocemente. O objetivo do tratamento fisioterapêutico é oferecer oportunidades e possibilidades adequadas para a criança aprender a interagir e a explorar o ambiente com mais funcionalidade e independência ${ }^{4}$. Essa intervenção pode ser direcionada para crianças com diagnóstico de paralisia cerebral, definida como distúrbio do movimento e da postura decorrente de uma lesão não progressiva que ocorre durante o período de desenvolvimento cerebral, e é adquirida antes dos dois primeiros anos de vida ${ }^{5}$.

A criança com paralisia cerebral diplégica apresenta comprometimento nos quatro segmentos do corpo; entretanto, os membros inferiores são mais afetados que os superiores. A distribuição da espasticidade é mais ou menos simétrica. As crianças geralmente possuem um bom controle de cabeça e um comprometimento de moderado a leve nos membros superiores. O estrabismo está presente em certo número de crianças e a fala normalmente não é afetada ${ }^{6}$.

Todas as crianças diplégicas possuem espasticidade, que é o aumento do tônus, causado pela lesão do neurônio motor superior, que pode ser observada com o crescimento da velocidade do movimento passivo. A espasticidade, que faz com que os movimentos da criança diminuam, apresenta as seguintes características: hiperreflexia, fraqueza muscular, clônus, padrões motores anormais, diminuição da destreza, pobre estabilidade postural, flexão dos membros superiores e extensão dos membros inferiores. Como a espasticidade é predominante em alguns grupos musculares e não em outros, as deformidades articulares aparecem com freqüência. De acordo com a gravidade da deficiência, sob o ponto de vista funcional, podem ser classificadas em cinco níveis (I, II, III, IV e V) ${ }^{7}$.
Segundo Kleinert e Effgen ${ }^{1}$, no nível I, a criança anda sem restrições e com limitações nas atividades motoras grossas mais sofisticadas; no nível II, locomove-se sem equipamento de auxílio e com limitações na marcha; no nível III, com equipamento de auxílio e limitações na marcha; no nível IV, possui mobilidade própria com restrições, sendo carregada, ou utiliza dispositivos motorizados em ambiente externo ou na comunidade; no nível $\mathrm{V}$, apresenta mobilidade seriamente limitada, mesmo com uso de tecnologia de assistência.

Crianças com paralisia cerebral podem apresentar alteração nos sistemas músculoesquelético, neuromotor, sensorial, gastrointestinal, cardiorrespiratório, cognitivo e comportamental; por isso, é importante avaliá-las como um todo, identificando suas capacidades e limitações funcionais e os sistemas que estão interferindo na execução de suas funções motoras para, posteriormente, intervir adequadamente em suas deficiências ${ }^{1}$.

Crianças com dez anos de idade estão aptas a desenvolver habilidades como pular corda, correr com facilidade, subir e descer escada sem apoio das mãos e alternando os pés, saltar, andar de bicicleta, chutar e arremessar bolas grandes ${ }^{8}$. Crianças com paralisia cerebral diplégica nesta idade apresentam padrão típico de flexão, rotação interna e adução do quadril, flexão dos joelhos e pé eqüino e em rotação interna, levando a outras compensações corporais, lordose lombar/escoliose que prejudicam o desenvolvimento de outras habilidades como levantar e andar6.

A postura típica, conseqüente da hipertonia e da espasticidade, dificulta a realização de uma marcha normal, o que possivelmente influencia nos picos de força, na capacidade de amortecimento dos membros, fazendo-os cair, de forma abrupta, sobre o solo9. A repetição sucessiva de um movimento faz com que a criança adquira percepção do estímulo e aprenda a responder de acordo com suas capacidades motoras $^{8}$. É por meio das tentativas de respostas, com erros e sucessos, que a criança irá processar as 
informações e aprender as atividades propostas $^{10}$. Para isso, é importante que o terapeuta proporcione possibilidades adequadas à criança para que possa desenvolver novas estratégias de exploração do ambiente.

Uma história detalhada de todo o desenvolvimento pré-natal e pós-natal da criança (colhida na anamnese) e uma avaliação qualitativa mensal são importantes para afastar a possibilidade de a criança perder habilidades presentes ${ }^{11}$. Outra forma de avaliar as capacidades motoras da criança é pela avaliação quantitativa, com a utilização de instrumentos de avaliação padronizados para crianças com paralisia cerebral, como a Gross Motor Function Measure ${ }^{12}$.

O objetivo, neste estudo, foi verificar a influência da intervenção fisioterapêutica na habilidade motora grossa de crianças com paralisia cerebral diplégica - nível II (marcha independente com limitações), pelo método Gross Motor Function Measure (GMFM) (avaliação quantitativa) e pela análise descritiva postural (avaliação qualitativa) nas habilidades de ficar sentado, em pé, andar, correr e pular.

\section{METODOLOGIA}

\section{Participantes}

Neste estudo, foram avaliadas duas crianças do sexo masculino, com idade média de dez anos, com diagnóstico de paralisia cerebral diplégica espástica, selecionadas de forma não aleatória, com base em critérios de inclusão previamente determinados.

Os critérios utilizados para inclusão foram crianças com diagnóstico de paralisia cerebral diplégica espástica, na faixa etária de dez anos, e freqüentadores assíduos da Clínica de Fisioterapia do Centro Universitário de Araraquara (Uniara), com marcha independente (grau de severidade leve) e com potencial para melhora no seu desempenho motor. $O$ critério para classificar a severidade das crianças com paralisia cerebral diplégica baseou-se no meio de locomoção, sendo esta independente e classificada como leve.
Foram excluídas deste estudo crianças que apresentaram distúrbios associados, tais como retardo mental, epilepsia e problemas ortopédicos como pé torto congênito e escoliose severa.

Durante este estudo, os pacientes não foram submetidos a qualquer outro tipo de intervenção ortopédica (cirurgias) e/ou medicamentosa (Toxina Botulínica).

\section{Materiais e métodos}

As crianças foram submetidas a uma avaliação clínica para coleta de dados pessoais e anamnese e, em seguida, ao teste $\mathrm{GMFM}^{12}$ e à análise descritiva postural. Estas avaliações foram realizadas no início e no final do estudo, ou seja, em um intervalo de quatro meses.

\section{Avvaliação quantitativa e qualitativa da habilidade motora grossa}

Para avaliação quantitativa da função motora grossa foi utilizado o método de GMFM0 ${ }^{12} \mathrm{e}$, na qualitativa, utilizou-se o recurso de captação de imagens em vídeo seguida de análise descritiva postural em uma ficha de avaliação nas posturas sentada no chão e em pé e nas funções andar, correr e pular.

\section{Método de intervenção fisioterapêutica}

O tratamento baseou-se no método neuroevolutivo criado por Beta Bobath e Karel Bobath, que tem como filosofia melhorar a funcionalidade de pacientes com lesão do sistema nervoso central, integrando os sistemas orgânicos. Além desse método, foram utilizadas técnicas cinesioterápicas que visam melhorar a função motora e a eficácia da marcha e integração sensorial para integrar os sistemas do organismo, favorecendo o desenvolvimento das habilidades motoras deficitárias. O tratamento foi realizado de forma lúdica, motivando as crianças a realizar atividades que exijam coordenação motora, equilíbrio, agilidade, destreza, além da correção postural, mobilizações e alongamentos. 
Foram utilizados, durante a intervenção, rolo pequeno e grande, banco de várias alturas, rampa, escada, prancha uniplanar e multiplanar, bola suíça grande e pequena, faixa elástica (bandagens), colchonete, esteira ergométrica, bastão, espaldar, cama elástica, brinquedos (bola de futebol, bolas pequenas, boliche, balde pequeno, carrinho, bambolê, amarelinha e tabuleiro de jogos), tornozeleira de $400 \mathrm{~g}$ e fita adesiva para fazer demarcações no chão.

Após serem identificadas as limitações funcionais pelo método GMFM, pela análise descritiva das posturas e pela funcionalidade das filmagens, foram traçados os seguintes objetivos para o tratamento:

- Passar de sentado para em pé, e vice-versa, em banco pequeno, sem apoio;

- Passar de sentado no chão para em pé, sem apoio;

- Na posição sentada, pegar objetos realizando flexão, rotação, inclinação e extensão de tronco;

- Sentar no chão, de lado;

- Passar de deitado no chão para sentado;

- Passar de semi-ajoelhado para em pé, sem usar os braços;

- Em pé, agachar para pegar objetos do chão, sem apoio;

- Manter-se na posição unipodálica;

- Andar em linha reta para frente, para trás e de lado;

- Andar em linha sinuosa;

- Andar segurando uma bola grande;

- Pular obstáculos em diferentes alturas, sem apoio;

- Andar com os olhos vendados;

- Correr;

- Pular dentro de um círculo;

- Pular na cama elástica, com apoio;

- Equilibrar-se em prancha uniplanar e skate, com apoio;

- Subir e descer escadas sem apoio, alternando os pés;

- Chutar bola;

- Empurrar bola contra resistência;

- Arremessar bola (vôlei);
- Apreensão de objetos;

- Subir de frente e descer de costas em rolo declinado, sem apoio de membros superiores, com auxílio;

- Deitado em decúbito ventral sobre skate, locomover-se com membros superiores;

- Sentado com membros inferiores flexionados e abduzidos (posição borboleta) sobre o skate; enquanto o terapeuta o empurra;

- Subir e descer espaldar;

Com base nas limitações funcionais observadas nas avaliações, foram estabelecidos objetivos funcionais diários, isto é, em cada sessão era designada uma habilidade funcional a ser predominantemente trabalhada.

Segundo Campos ${ }^{13}$, as sessões eram conduzidas de acordo com a seguinte estrutura:

- Função: habilidade funcional específica;

- Pré-teste: observação do desempenho da criança na habilidade funcional escolhida;

- Conduta terapêutica: realização de atividades, visando ao aprimoramento da habilidade funcional determinada no dia;

- Pós-teste: observação de desempenho da criança, pós-intervenção, na mesma habilidade funcional.

A intervenção fisioterapêutica foi realizada com freqüência de duas vezes por semana, com duração de 50 minutos, totalizando 32 sessões durante quatro meses.

A Tabela 1 descreve as atividades realizadas de acordo com a habilidade funcional visada.

Ao término do tratamento, foi realizada a pós-avaliação, seguindo os mesmos critérios da primeira avaliação.

\section{Análise dos resultados}

Após ser calculada a média do escore percentual total de cada dimensão, foi realizada uma análise descritiva das avaliações pelo método GMFM, antes e após a intervenção fisioterapêutica. 
Tabela l: Apresentação das atividades realizadas em cada uma das habilidades funcionais. Araraquara, 2007

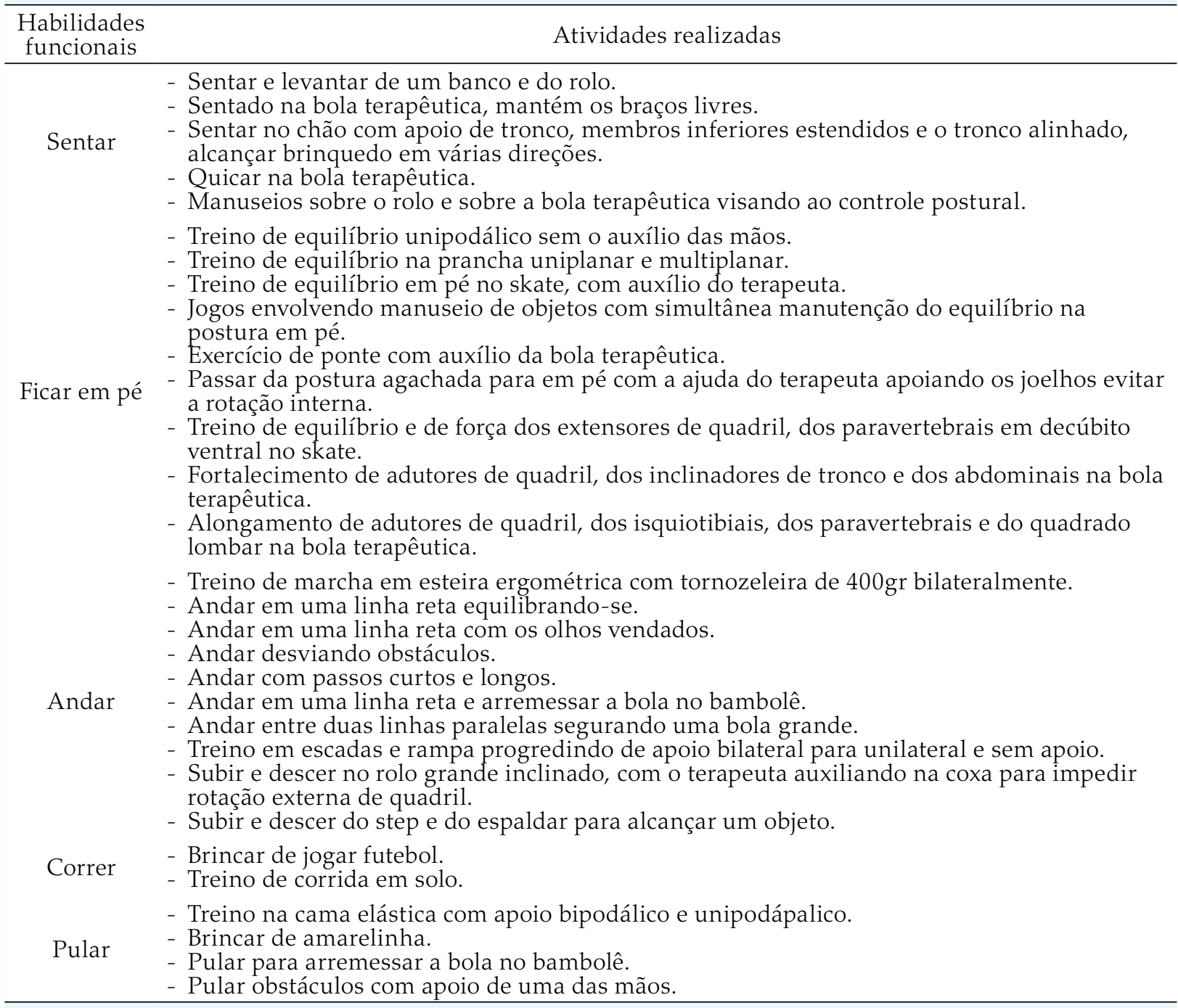

\section{RESULTADOS}

\section{Análise quantitativa pelo método Gross Motor Function Measure}

A Figura 1 mostra os resultados percentuais da pré e pós-avaliação nas Dimensões B, D e E dos participantes.

Pode-se observar que, na pré e pós-avaliação da Dimensão B (sentar), o desempenho motor dos participantes foi melhor em relação ao verificado nas outras dimensões. Na D (ficar em pé), não houve grandes modificações da pré para a pós-avaliação no desempenho dos participantes; entretanto, foi na Dimensão E (andar, correr e

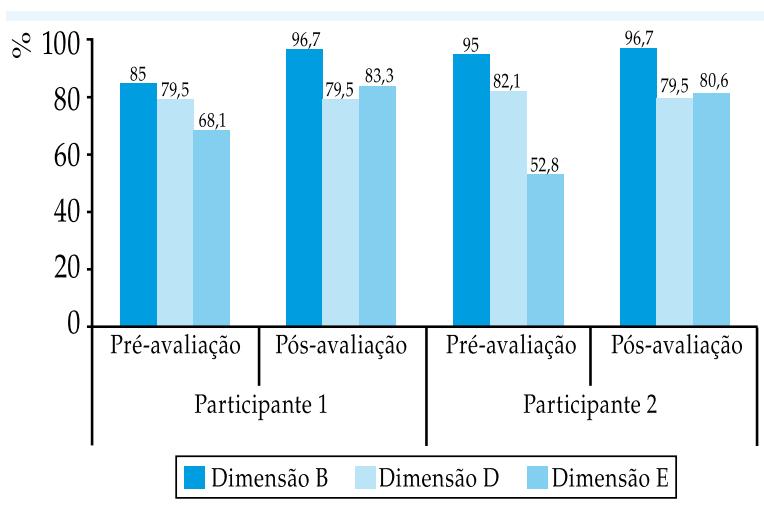

Figura 1: Valores percentuais das habilidades motoras nas Dimensões B, D e E obtidas na pré e pós-avaliação pelo método GMFM 
pular) que eles apresentaram maior ganho motor com a intervenção fisioterapêutica.

Com base nos resultados observados, após intervenção fisioterapêutica, tanto da avaliação quantitativa (GMFM) quanto da qualitativa (análise descritiva postural), verificou-se que o participante 1 foi capaz de realizar os itens 28 (sentada de lado (D): mantém braços livres, 5 segundos) e 29 (sentada de lado (E): mantém braços livres, 5 segundos) da Dimensão B (sentar), conseguindo manter-se sentado de lado com apoio de uma das mãos, além de ter apresentado melhora qualitativa em sua postura sentada no chão. Na Dimensão D (ficar em pé), mantiveram-se os resultados quantitativos obtidos pelo método GMFM, porém observou-se melhora na simetria postural. Na Dimensão E (andar, correr e pular), após a intervenção, a criança é capaz de realizar o item 73 (Em pé: anda para frente 10 passos consecutivos entre linhas paralelas afastadas em $20,32 \mathrm{~cm}$ ), conseguindo andar 10 passos consecutivos entre linhas paralelas. Nas tarefas de correr, o participante tinha atingido a pontuação máxima na pré-avaliação. No item 82 (em pé sobre o pé direito - apoio unipodal): salta com o pé direito 10 vezes dentro de um círculo de $61 \mathrm{~cm}$ de diâmetro) e no 83 (em pé sobre o pé esquerdo - apoio unipodal): salta com o pé esquerdo 10 vezes dentro de um círculo de $61 \mathrm{~cm}$ de diâmetro), os resultados foram mantidos, pois o participante tinha dificuldade de ficar em apoio unipodálico; entretanto, melhorou qualitativamente na fase de impulsão com os dois pés simultâneos e na de amortecimento. O participante 2, na pós-avaliação, mostrou que era capaz de realizar os itens 28 (sentado de lado - direito): mantém, braços livres, 5 segundos) e 29 (sentado de lado - esquerdo): mantém braços livres, 5 segundos), da Dimensão B (sentar), conseguindo ficar mais tempo na posição de sentar de lado com apoio de uma das mãos, além de apresentar melhora qualitativa em sua postura. Na Dimensão D (ficar em pé), no item 62 (Em pé: abaixa para sentar-se no chão com controle, braços livres), o participante regrediu passando da função (abaixa-se para sentar-se no chão com controle, braços livres) para (abaixa-se para sentar-se no chão com controle, usando braço(s) ou segurando-se). Isso provavelmente ocorreu porque diminuiu a rotação interna, o que desestabilizou o controle para abaixar sem apoio das mãos. Na Dimensão E, o participante aprimorou a tarefa do item 73 , andando para frente com dez passos consecutivos entre linhas paralelas afastadas. Nos itens 82 e 83, os participantes mantiveram o escore 0 (não iniciam o saltar com o pé direito e esquerdo, respectivamente, porém saltam com os dois pés simultaneamente). No item 88 , não conseguia pular um degrau com os dois pés, mas, após a intervenção, realizou essa tarefa saltando com os dois pés simultaneamente. Na análise qualitativa, o participante apresentou melhora na impulsão, na queda, no equilíbrio e na coordenação.

\section{Anớlise qualitativa pela descrição das posturas estáticas (sentada no chão e em pé) e dinômicas (andar, correr e pular) do participante 1}

Na postura sentada no chão, a posição da cabeça evoluiu de anteriorizada e fletida para neutra, e os ombros, cotovelos, punhos e dedos, de semifletidos para posição neutra e relaxados. $\mathrm{O}$ tronco, que se encontrava com hipercifose torácica e hiperlordose lombar, apresentou curvas fisiológicas. A pelve no sacro melhorou a anteversão, permanecendo com leve retroversão. Os quadris, de semifletidos, aduzidos e rodados internamente evoluíram para posição neutra. Os joelhos semifletidos diminuíram a flexão. Os tornozelos de flexão plantar e inversão evoluíram para leve inversão, e artelhos passaram de fletidos para a posição neutra.

Na postura em pé, melhorou a posição da cabeça, evoluindo de anteriorizada e inclinada à direita para posição simétrica; os ombros evoluíram de aduzidos e rodados internamente para aduzidos com leve rotação interna; os cotovelos, de semifletidos para estendidos e membros superiores relaxados, e os punhos e dedos fletidos demonstraram posição neutra. $\mathrm{O}$ tronco semifletido apresentou posição neutra. Os quadris, de semifletidos, aduzidos e rodados internamente, evo- 
luíram para estendidos. Os tornozelos direito e esquerdo, de evertidos passaram à posição neutra e leve eversão, respectivamente; os pés, de aduzidos para abduzidos em uma postura relaxada, e os dedos, de fletidos para semifletidos.

Nas funções dinâmicas andar, correr e pular, além da evolução qualitativa postural, observou-se melhora no equilíbrio, força de impulsão, coordenação, velocidade e ritmo durante a execução dos movimentos.

\section{Annólise qualitativa pela descrição das posturas estáticas (sentada no chão e em pé) e dinâmicas (andar, correr e pular) do participante 2}

Na postura sentada no chão, a posição da cabeça evoluiu de anteriorizada para levemente anteriorizada; os ombros, de semifletidos e semi-abduzidos para semifletidos; os cotovelos, de fletidos para semifletidos; os punhos, de estendidos para semifletidos, e os dedos permaneceram semifletidos. $\mathrm{O}$ tronco de hipercifose torácica e lombar retificada apresentou curvas fisiológicas. A pelve, de retrovertida (sentada no sacro) para posição neutra. Os quadris, de semifletidos e rodados externamente evoluíram para semifletidos. Os joelhos permaneceram na mesma posição, ou seja, em semiflexão. Os tornozelos, de flexão plantar para dorsiflexão e os artelhos, da posição neutra para estendidos.

Na postura em pé, a posição da cabeça passou de semifletida para levemente inclinada à direita; os ombros, de leve abdução e pequena rotação interna para posição neutra. Os cotovelos permaneceram semifletidos. Os punhos mantiveram-se na posição neutra. Os dedos passaram de semifletidos para estendidos; o tronco evoluiu de leve inclinação lateral direita para posição neutra. Os quadris passaram de semifletidos para posição neutra, e os joelhos, de semifletidos para posição neutra. Os tornozelos direito e esquerdo que se encontravam evertidos passaram para posição neutra e leve eversão, respectivamente. Os pés de abduzidos evoluíram para leve abdução. Os artelhos passaram de fletidos para estendidos.
Nas funções dinâmicas, andar, correr e pular, além da evolução qualitativa postural, observou-se o seguinte quadro: melhora no equilíbrio, força na impulsão, coordenação, velocidade e ritmo durante a execução dos movimentos.

\section{DISCUSSÃO}

Os resultados deste estudo fornecem informações sobre a influência da intervenção fisioterapêutica e a utilização de técnicas específicas, capazes de avaliar, de forma quantitativa e qualitativa, a evolução da habilidade motora grossa de crianças com paralisia cerebral diplégica.

Após a intervenção fisioterapêutica, verificaram-se, por meio da avaliação quantitativa pelo método GMFM, resultados percentuais positivos para ambos participantes, como mostra a Figura 1. Esses resultados estão de acordo com os estudos de Harries ${ }^{14}$, que examinou a função motora grossa de crianças com paralisia cerebral tetraplégica, de uma escola de educação especial, e de outras com desenvolvimento normal de três a oito anos de idade, que, durante o período deste estudo, tiveram os escores do GMFM melhorados de acordo com o grau de severidade de cada criança, atingindo um platô na idade de seis a sete anos. Este estudo demonstrou que as mudanças no desenvolvimento da função motora grossa dessa população foram semelhantes às do processo de desenvolvimento das crianças normalmente desenvolvidas.

O programa de intervenção fisioterapêutica aprimorou as habilidades funcionais dos participantes nos aspectos qualitativos da função motora grossa, como o sentar, o ficar em pé, o andar, o correr e o pular. Esse resultado foi observado por Mota e Pereira ${ }^{15}$, que verificaram, por meio da intervenção fisioterapêutica realizada em pacientes com diagnóstico clínico de paralisia cerebral com hemiparesia espástica, na faixa etária de cinco a 11 anos de idade, melhora no quadro motor em relação ao equilíbrio e à coordenação motora.

Com base nos resultados observados, após intervenção fisioterapêutica, da avaliação quantitativa (GMFM) e da qualitativa (análise descritiva postural) dos participantes, verificaram-se valo- 
res mais altos na Dimensão B do que nas D e E. Segundo Ozu e Galvão ${ }^{16}$, em um paciente com paralisia cerebral diplégica espástica, espera-se que as maiores dificuldades observadas com a avaliação pelo GMFM sejam nas dimensões finais D e E, pois exigem maior controle do tronco, pelve e membros inferiores para realizar as tarefas de ficar em pé, andar, correr e pular. Portanto, segundo o mesmo autor, a área-meta em que deveriam demonstrar melhores resultados após a intervenção seriam as dimensões D e E.

No entanto, o programa de intervenção fisioterapêutica propiciou aprimoramento da habilidade motora grossa, principalmente nas Dimensões B e E. Na D, os participantes não apresentaram grandes avanços na posição em pé, pela pequena base de suporte que demonstraram nessa postura. Segundo Bobath ${ }^{6}$, a base de apoio das crianças diplégicas, na posição ortostática e durante a marcha, é pequena, o que interfere no equilíbrio postural. Umphred ${ }^{17}$ ressalta que a hipertonia espástica impede uma troca homogênea entre a mobilidade e a estabilidade do corpo, o que poderia explicar a grande dificuldade para a aquisição da habilidade motora na postura estática. Latash ${ }^{18}$ destaca ainda que, para obter controle postural e, conseqüentemente, equilíbrio estático, é necessário um ajuste fino e adequado entre as diversas articulações corporais.

A Dimensão E (andar, correr e pular) envolve posturas dinâmicas e equilíbrio dinâmico, e foi nessa dimensão que os participantes apresentaram grandes avanços no desempenho das atividades. A melhora do equilíbrio, da força e da coordenação observada na análise qualitativa após intervenção está de acordo com o estudo de Teive et al. ${ }^{19}$, ao relatarem que a fisioterapia pode prover condições que facilitem o controle do tônus, contribuindo para ajudar na aquisição de posturas e movimentos dinâmicos.

Os dados quantitativos, obtidos com o GMFM, e os qualitativos, pela descrição postural, auxiliaram nas tomadas de decisão quanto ao planejamento da intervenção, e na visualização do real desenvolvimento e desempenho dos participantes nas habilidades motoras grossas.
Os resultados obtidos da evolução das habilidades que envolvem diretamente as capacidades motoras após intervenção fisioterapêutica, pelo método neuroevolutivo Bobath, estão de acordo com Knox e Evans ${ }^{20}$, que pesquisaram os efeitos funcionais da terapia Bobath em crianças com paralisia cerebral - diplégicas, quadriplégicas espásticas e quadriplégicas atetóides. Verificou-se que os participantes tiveram ganhos na função motora e nos cuidados pessoais após serem submetidos a essa terapia.

A eficácia da intervenção fisioterapêutica nos dois participantes deste estudo reflete-se na melhora da qualidade de vida para realizar atividades de vida diária sem muito gasto energético. No estudo de Damiano e $\mathrm{Abel}^{21}$, verificou-se, após o treino de força em crianças com paralisia cerebral diplégica e hemiplégica espástica, aumento significativo na velocidade e no ritmo na Dimensão E (andar, correr e pular) pelo método Gross Motor Function Measure.

Provavelmente, o uso de técnicas cinesioterápicas, como esteira ergométrica, utilizadas durante a intervenção fisioterapêutica deste estudo, favoreceu a melhora da função motora e a eficácia da marcha. Tais achados coincidem com o estudo de Bonamido et al. ${ }^{2}$ que observaram melhora na função motora e na eficácia da marcha em pacientes diplégicos, após o treinamento da marcha em esteira ergométrica, tendo os indivíduos referido maior independência no exercício.

O tratamento fisioterapêutico, realizado de forma lúdica, provavelmente tenha motivado as crianças a se empenhar em outros tipos de atividades que exijam coordenação motora, equilíbrio, agilidade e destreza, além da correção postural, mobilizações e alongamentos. $\mathrm{O}$ desenvolvimento dessas habilidades consiste em treinar crianças com paralisia cerebral para desempenhar, de forma independente, as atividades essenciais à sua vida diária ${ }^{23}$.

De acordo com Lorenzine ${ }^{24}$, a brincadeira simples e criativa pode ajudar no processo de aquisição da habilidade motora. Nesse contexto, o movimento é uma brincadeira que o ser humano desenvolve desde o nascimento e lhe proporciona 
uma troca com o meio em que vive, adquirindo novas experiências. Portanto, tal atividade é um instrumento de suma importância, pois fornece à criança experiências para seu desenvolvimento sensorial, motor, perceptual, cognitivo, afetivo e cultural, inclusive daquela portadora de deficiência motora.

Uma observação a ser feita para trabalhos futuros com relação à bibliografia existente é que alguns deles não especificam os itens do método em que a criança apresenta melhora, mas destacam apenas a evolução ou não do paciente, representada pelo escore total. Ficaria mais detalhado se os estudos demonstrassem em que item o paciente evoluiu.

\section{CONCLUSÃO}

A intervenção fisioterapêutica, com base no conceito neuroevolutivo Bobath e no tratamento cinesioterápico, associada às atividades lúdicas, pode contribuir para a evolução quantitativa e o refinamento qualitativo do desempenho funcional dos participantes com paralisia cerebral diplégica espástica, na habilidade motora grossa.

\section{REFERÊNCIASS}

1. Kleinert Jor, Effgen. Intervenção Precoce. Fisioterapia Pediátrica: atendendo às necessidades das crianças. Effgen, SK. [traduzido por Eliane Ferreira]. Rio de Janeiro: Guanabara Koogan; 2007.

2. Bergeron K, Dichter CG. Estudo de Caso: síndrome de Down. Fisioterapia Pediátrica: atendendo às necessidades das crianças. Effgen, SK. [traduzido por Eliane Ferreira]. Rio de Janeiro: Guanabara Koogan, 2007.

3. Brossman Hl, Schrepfer C. Estudo de Caso: fibrose cística. Fisioterapia Pediátrica: atendendo às necessidades das crianças. Effgen, SK. [traduzido por Eliane Ferreira]. Rio de Janeiro: Guanabara Koogan, 2007.

4. Pereira K, Tudella E. Fisioterapia: orientação aos pais para estimulação sensório-motora de crianças com síndrome de Down. Santana, V.D. Síndrome de Down. [livro em preparação].
5. Schwartzman JS. Paralisia Cerebral. Arquivos Brasileiros de Paralisia Cerebral;2004;1(1):4-17.

6. Bobath, Bobath. Desenvolvimento motor nos diferentes tipos de Paralisia Cerebral. $1^{a}$ Ed. São Paulo:Manole;1989.

7. Pereira CLG, Fontanetti S, Lopes DV. Paralisia Cerebral. [acesso:em 18 nov 2007]. Disponível em: http://www.fisioneuro.com.br/ver_pesquisa. php?id=1.

8. Gallahue DL, Ozmun JC. Compreendendo o desenvolvimento motor:bebês, crianças, adolescentes e adultos. $2^{\text {a }}$ ed., São Paulo:Phorte Editora;2003.

9. Filippin NT, Bonamigo ECB. Implicações terapêuticas da análise dinâmica da marcha na paralisia cerebral diplégica - um estudo de caso. Praxisterapia, Julho. 2003;8(8):25-36.

10. Magill RA. Aprendizagem Motora:conceitos e aplicações. $5^{\text {a }}$ ed. São Paulo:Blücher;2000.

11. Russman BS, Ashwal S. Evaluation of the Child with Cerebral Palsy. Seminars in Pediatric Neurology. March. 2004;11(1):47-57.

12. Russell D, Rosenbaum P, Growland C, Hardy S, Lane M, Plews N, Mcgavin H, Cadman D and Jarvis S. Administration and Scoring. In:Gross Motor Function Measure Manual. Second Edition. Toronto: McMaster University; Set 1993.

13. Campos AC, Santos NSA, Tudella E, Pereira K, Rocha NAC. F. Impacto de programa fisioterapêutico no desempenho funcional da criança com doença de Charcot-Marie-Tooth tipo 2:estudo de caso. Revista Fisioterapia e Pesquisa da Universidade de São Paulo. Maio/Agosto 2007;14(2):77-83.

14. Harries N, Kassirer M, Amichai T, Lahat E. Changes over years in gross motor function of 3-8 years old children with cerebral palsy:using the Gross Motor Function Measure (GMFM - 88). Isr Med Assoc. J. 2004;7;6:408-411.

15. Mota AP, Pereira Js. Influência da fisioterapia nas alterações motoras em crianças com paralisia cerebral. Fisioterapia Brasil. Maio/junho. 2006;7(3):209-212.

16. Ozu MHU, Galvão MCS. Fisioterapia na Paralisia Cerebral. In: Moura EW, Silva PAC. Fisioterapia: aspectos clínicos e práticos da reabilitação. $2^{\mathrm{a}}$ ed. São Paulo: Artes Médicas; 2005.

17. Umphred DA. Fisioterapia Neurológica. Manole:São Paulo;1994. 
18. Latash M. Strategies of motor rehabilitation:the role of adaptative changes_Anais do III Congresso Internacional de Reabilitação Motora. 1998. Águas de Lindóia, São Paulo, publicado na Revista Brasileira de Fisioterapia; 1998;2(15).

19. Teive HAG, Zonta M, Kumagai Y. Tratamento da espasticidade:uma atualização. Arq Neuropsiquiatr, 1998;56(4):852-858

20. Knox V, Evans AL. Evaluation of the functional effects of a course of Bobath therapy in children with cerebral palsy:a preliminary study. Dev Med Child Neurol. Jul 2002;44(7):447-60. [acesso em 2007 dec. 15] Disponível em: http://www.proquest.com.
21. Damiano DL, Abel MF. Functional outcomes of strength-training of functional ability and walking efficiency in adolescents with cerebral palsy. Dev Med Child Neurol 1995;37:763-775.

22. Bonamido ECB;Canavesi A, Plentz RD;Biarski $\mathrm{B}$, Gotardo PC. Treino intensivo no contexto da tarefa - uma nova abordagem para a reabilitação de pacientes com seqüela de paralisia cerebral. Praxisterapia. Dezembro. 5(5):34-46.

23. Shepherd RB. Fisioterapia em pediatria. $3^{a}$ ed. São Paulo: Santos;1996.

24. Lorenzine MV. Brincando a brincadeira com a criança deficiente: novos rumos terapêuticos, $1^{\text {a }}$ ed. São Paulo: Manole; 2002. 